Reprod. Nutr. Dévelop., 1983, 23 (2 B), 437-442.

\title{
Effets à long terme de l'insuline sur la conversion adipocytaire des cellules de la lignée Ob17
}

\author{
P. GRIMALDI, P. DJIAN, R. NEGREL, G. AILHAUD
}

Centre de Biochimie du CNRS, (LP 7300), Faculté des Sciences, Parc Valrose, 06034 Nice Cedex, France.

Summary. Long-term effects of insulin on the conversion of cells of the $\mathrm{Ob} 17$ line into adipocytes.

The Ob17 cell line was established from the epididymal fat pad of ob/ob mice. Chronic exposure to physiological concentrations of insulin led to a net increase in lipogenic enzyme activities $\left(\mathrm{EC}_{50}=1\right.$ to $\left.3 \mathrm{nM}\right)$. Proinsulin exerted the same effects at higher concentrations $\left(E C_{50}=10\right.$ to $\left.30 \mathrm{nM}\right)$. The mitogenic effects of both hormones appeared only at supraphysiological concentrations $\left(\mathrm{EC}_{50}=300 \mathrm{nM}\right)$. We conclude this was a net dissociation between the lipogenic and mitogenic effects of insulin during differentiation of the Ob17 cells.

\section{Introduction.}

La lignée préadipocytaire Ob17 a été établie en 1978 à partir d'une fraction purifiée d'adipocytes de souris génétiquement obèse (C57 BL/6J (Négrel et al., 1978). Lors de la phase de multiplication, les cellules Ob17 présentent la morphologie et les caractéristiques enzymatiques de fibroblastes. Les cellules confluentes cessent dans un premier temps de se diviser, puis dans certaines zones privilégiées de la monocouche cellulaire se produisent des mitoses qualifiées de " mitoses post-confluentes ". Cette prolifération limitée dans le temps cesse après une semaine environ et conduit à la formation de colonies de cellules qui subissent la conversion adipocytaire. La formation de ces colonies est une étape importante pour conduire à une augmentation du nombre de cellules adipeuses par rapport à la population totale. Elle n'est cependant pas essentielle à la différenciation puisque le blocage de ces mitoses post-confluentes n'empêche pas la différenciation de se produire chez des cellules isolées (Djian et al., 1982). La formation des colonies de cellules adipeuses est sous la dépendance d'un facteur présent dans le sérum de veau fétal et qui a pu être mis également en évidence dans l'hypophyse de bœuf. Enfin, la triiodothyronine est nécessaire, à concentration physiologique, à l'expression du programme de différenciation des cellules Ob17 (Gri- 
maldi et al., 1982). L'ensemble du processus de conversion adipocytaire se déroule sur 2 à 3 semaines. II passe par l'induction spécifique des activités enzymatiques caractéristiques des adipocytes comme la lipoprotéine et la monoglycéride lipases qui apparaissent précocement alors que la glycérol-3-phosphate déshydrogénase, I'acide : CoA ligase, l'acide gras synthétase ou la lipase hormono-sensible sont des marqueurs plus tardifs de la différenciation.

Des études antérieures ont montré que les cellules Ob17 possèdent des récepteurs liant l'insuline avec une forte affinité $\left(K_{d}=10^{-9} \mathrm{M}\right)$. Le nombre de récepteurs est de 8000 sites par cellule et ne varie pas avec le degré de différenciation. D'autre part, l'exposition des cellules Ob17 à l'insuline conduit à une nette diminution du nombre des récepteurs à I'hormone (Grimaldi et al., 1979). Cette diminution de la capacité fixatrice s'accompagne de la diminution puis de la disparition de l'effet stimulateur de l'insuline sur le transport de l'acide $\alpha$-aminoisobutyrique (Ailhaud et al., 1979).

\section{Résultats.}

L'évolution, en fonction du temps de culture, des activités spécifiques de trois marqueurs de la différenciation, l'acide : CoA ligase (fig. 1A), l'acide gras synthétase (fig. 1B) et la glycérol-3-phosphate déshydrogénase (fig. 1C), a été suivie pour des cellules Ob17 maintenues depuis la confluence en l'absence ou en présence d'insuline (170 $\mathrm{nM}$ ). Dans un milieu de culture standard, c'est-à-dire contenant $10 \%$ de sérum de veau fétal, les activités de synthèse et d'activation des acides gras augmentent. L'augmentation de l'activité glycérol-3-phosphate

(A)

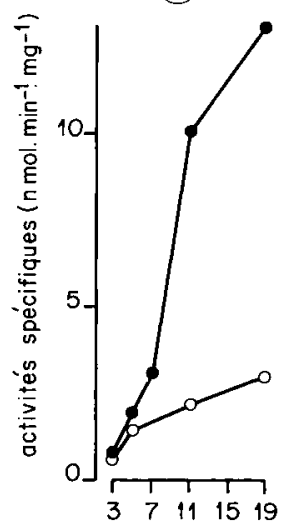

(B)

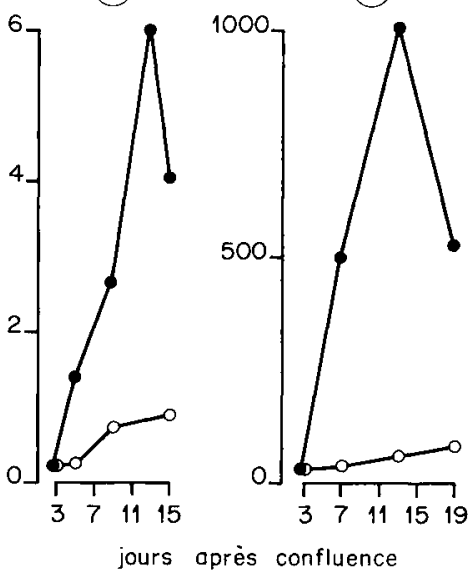

(D)

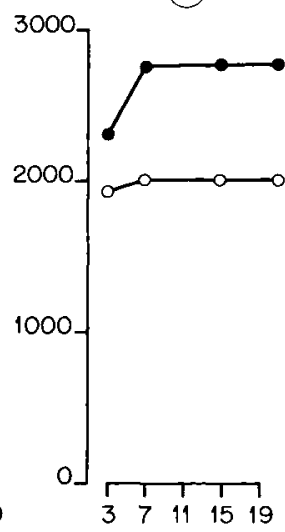

FIG. 1. - Effets de l'insuline sur la conversion adipocytaire des cellules Ob17. Les cellules sont cultivées à partir de la confluence (Jour 0) dans un milieu de culture enrichi en $T_{3}(1,5 \mathrm{nM})$ et en l'absence $(0-0)$ ou en présence (- - d d'insuline $(170 \mathrm{nM})$. Les activités enzymatiques sont déterminées aux jours indiqués et représentent la moyenne de deux déterminations. $\mathrm{A}=$ acide : CoA ligase, $\mathrm{B}=$ acide gras synthétase, $\mathrm{C}=$ glycérol-3-phosphate déshydrogénase, $\mathrm{D}=$ lactate déshydrogénase. 
déshydrogénase est moins forte mais est toutefois significative. Lorsque le milieu de culture est enrichi en insuline, les niveaux des activités lipogéniques augmentent très nettement après le cinquième jour de traitement. Ainsi par rapport à la condition standard, les activités sont 5 fois plus élevées pour l'acide : CoA ligase, 10 fois plus pour l'acide gras synthétase et 20 fois pour la glycérol-3phosphate déshydrogénase. L'activité de la lactate déshydrogénase (fig. 1D), enzyme qui ne participe pas à la lipogenèse, est déjà très élevée avant la différenciation et évolue peu en l'absence ou en présence d'insuline. Cette action amplificatrice de l'insuline sur la conversion adipocytaire des cellules Ob17 est identique à celle obtenue dans les mêmes conditions avec d'autres modèles préadipocytaires (3T3- $L_{1}$ ) (Green et Kehinde, 1975 ; Grimaldi et al., 1978).

Toutefois à concentration supraphysiologique, l'insuline possède sur beaucoup de modèles cellulaires un pouvoir mitogénique (King et al., 1980). Ces expériences ne pouvaient exclure une action mitogénique de I'hormone conduisant, lors de la formation des colonies adipeuses, à une augmentation du nombre de cellules susceptibles de se différencier. Cette hypothèse a été vérifiée en
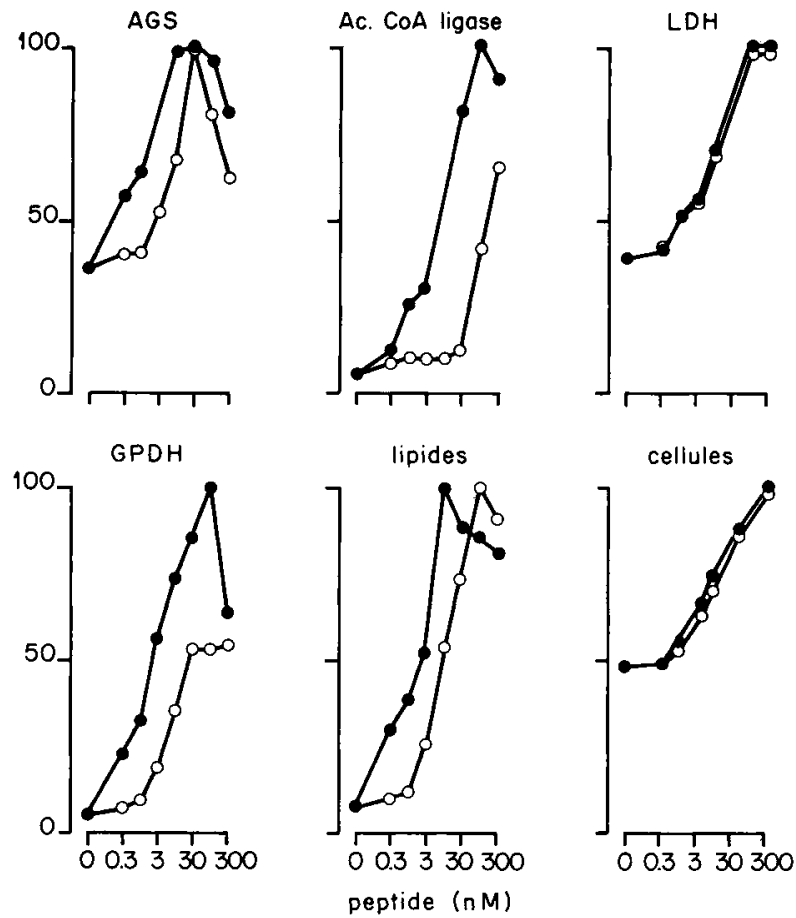

FIG. 2. - Effets lipogéniques et mitogéniques de l'insuline et de la proinsuline sur les cellules Ob17. A partir de la confluence, le milieu de culture est enrichi en insuline de porc ( $-\bullet)$ ou en proinsuline de porc $\left(O_{-} \mathrm{O}\right)$ à diverses concentrations. Les activités spécifiques de l'acide gras synthétase (AGS), de l'acide : CoA ligase, de la lactate déshydrogénase (LDH) et de la glycérol-3-phosphate déshydrogénase $(\mathrm{GPDH})$ ont été mesurées au $10^{\mathrm{e}}$ jour après confluence. Le nombre de cellules par boîte de culture et la quantité de triglycérides cellulaires ont été déterminés après 13 jours de traitement. Les résultats sont représentés en pourcentage de la réponse maximale à l'insuline. 
comparant les réponses mitogénique et lipogénique à l'insuline et à la proinsuline des cellules Ob17. Les effets à long terme de I'insuline et de la proinsuline de porc ont été examinés sur des cellules Ob17 exposées depuis la confluence à des concentrations variables en ces deux hormones (fig. 2). La réponse lipogénique aux deux hormones a été mesurée par les niveaux des activités spécifiques de trois marqueurs de la conversion adipocytaire après 10 jours de traitement (acide gras synthétase, acide : CoA ligase et glycérol-3-phosphate déshydrogénase) et par le dosage de la quantité de triglycérides accumulés par cellule 13 jours après confluence. Les effets mitogéniques de l'insuline et de la proinsuline ont été suivis par la détermination du nombre de cellules par boîte, 13 jours après confluence. L'activité de la lactate déshydrogénase - enzyme non impliquée dans la lipogenèse - a été également mesurée après 10 jours de traitement par les deux hormones. L'effet amplificateur de l'insuline sur l'apparition des marqueurs de différenciation s'exerce dans une zone de concentrations physiologiques, les concentrations de demi-effet pour les trois marqueurs de différenciation se situant entre 1 et $3 \mathrm{nM}$. L'exposition des cellules à la proinsuline conduit également à une nette amplification de l'expression des marqueurs de lipogenèse mais à des concentrations plus élevées $\left(\mathrm{EC}_{50}=6\right.$ à $\left.30 \mathrm{nM}\right)$. D'autre part, les niveaux maximums en glycérol-3-phosphate déshydrogénase et en acide : CoA ligase obtenus après traitement par la proinsuline sont inférieurs à ceux obtenus après traitement par l'insuline. Ces différences entre les potentialités des deux hormones ont pu être observées à divers moments du processus de conversion adipocytaire et pour d'autre marqueurs de la différenciation comme la diglycéride acyl-CoA-acyltransférase. L'effet lipogénique des deux hormones est également très net sur le contenu intracellulaire en triglycérides. L'exposition des cellules Ob17 pendant 13 jours à $10 \mathrm{nM}$ en insuline ou à $100 \mathrm{nM}$ en proinsuline conduit à une accumulation de triglycérides 15 fois plus forte que celle observée dans un milieu non enrichi en ces hormones. Les concentrations de demi-effet sont de $2 \mathrm{nM}$ pour l'insuline et de $10 \mathrm{nM}$ pour la proinsuline. La lactate déshydrogénase présente un

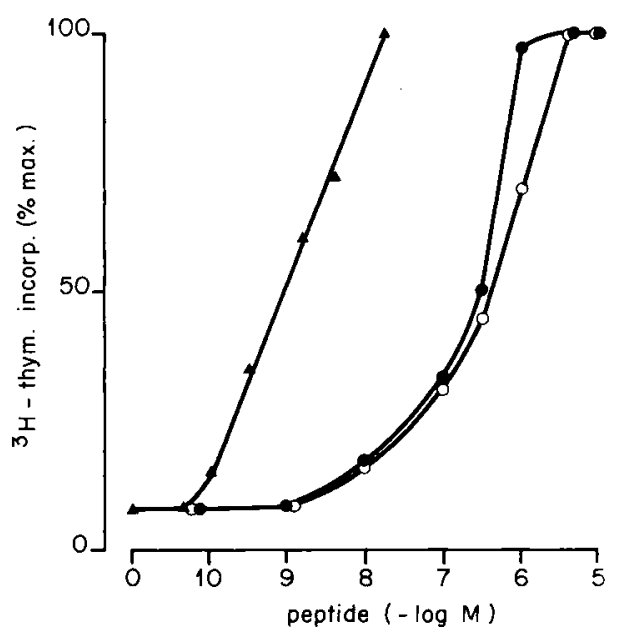

FIG. 3. - Réinitiation de la synthèse d'ADN par les cellules $O b 17$. Les cellules en phase de croissance sont placées pendant $24 \mathrm{~h}$ dans un milieu dépourvu de sérum. Le milieu de culture est alors supplémenté en insuline de porc $(-)$ ), en proinsuline de porc $(\mathrm{O}-\mathrm{l}$ ) ou en IGF-I ( $\mathbf{\Delta}-\mathbf{\Delta})$ à diverses concentrations et en thymidine tritiée $(3 \mu \mathrm{M}, 0,5 \mu \mathrm{Ci} / \mathrm{ml})$. Vingtquatre heures après, la quantité de radioactivité incorporée dans I'ADN cellulaire est déterminée. Les résultats sont représentés en pourcentage de la réponse maximale à l'insuline. 
statut particulier puisque les courbes dose-réponse obtenues pour les deux hormones sont superposables avec un $\mathrm{EC}_{50}$ de $10 \mathrm{nM}$.

Les effets mitogéniques à long terme de l'insuline et de la proinsuline se traduisent par une augmentation d'un facteur 2 du nombre de cellules par boîte de culture après 13 jours de traitement. Les concentrations de demi-effet pour les deux hormones sont supérieures à $10 \mathrm{nM}$. Afin de mesurer plus précisément les effets mitogéniques des deux hormones, des expériences de réinitiation de la synthèse d'ADN ont été réalisées. Pour ces expériences, rapportées dans la figure 3 , les cellules $0 b 17$ en phase exponentielle de croissance ont été rendues quiescentes par une incubation de $24 \mathrm{~h}$ en milieu de culture dépourvu de sérum. Les cellules sont alors placées pendant $24 \mathrm{~h}$ dans un milieu de culture contenant diverses concentrations en insuline, en proinsuline ou en " insulin-like growth factor-I " (IGF-I) et de la thymidine $(3 \mu \mathrm{M}, 0,5 \mu \mathrm{Ci} / \mathrm{ml})$. Dans ces conditions, les concentrations de demi-effet sont de $300 \mathrm{nM}$ pour l'insuline et de $500 \mathrm{nM}$ pour la proinsuline. L'amplitude de la réponse est identique pour les deux hormones. IGF-I agit à une concentration beaucoup plus faible, la demie réponse maximale à l'insuline étant obtenue à $1 \mathrm{nM}$ en ce facteur.

\section{Conclusion.}

En conclusion, l'insuline joue pendant la conversion adipocytaire des cellules Ob17 un rôle modulateur de l'expression du programme de différenciation. Cette modulation s'effectue dans des zones de concentrations physiologiques en hormone. Le modèle cellulaire constitué par les cellules Ob17 a permis pour la première fois la séparation des effets mitogéniques et lipogéniques de l'insuline. Les valeurs $d^{\prime} E C_{50}$ pour les effets lipogéniques et le décalage existant entre les courbes dose-réponse pour l'insuline et la proinsuline sont en accord avec l'affinité et la spécificité des récepteurs à l'insuline des cellules Ob17. Par contre les hautes concentrations en insuline ou en proinsuline nécessaires pour promouvoir la croissance des cellules semblent indiquer que ces effets mitogéniques mettent en jeu les récepteurs des " insulin-like growth factors». Ces résultats permettent d'exclure une éventuelle action mitogénique de l'insuline sur les mitoses postconfluentes qui conduisent à la formation des colonies de cellules adipeuses.

La lignée préadipocytaire Ob17 constitue un modèle de choix pour l'étude des mécanismes moléculaires impliqués dans les réponses à long terme à l'insuline.

8e Réunion du groupe Développement I.N.R.A., Tours, 12-13 mai 1982.

\section{Références}

AILHAUD G., GRIMALDI P., NEGREL R., SERRERO G., VERRANDO P., 1979. Multiplication and differentiation of preadipocyte cell lines isolated from mouse epididymal fat pad. In AILHAUD G., Obesity : cellular and molecular aspects. Coll. INSERM, vol. 87, 51-64.

DJIAN P., GRIMALDI P., NEGREL R., AILHAUD G., 1982. Adipose conversion of Ob17 preadipocytes $=$ relationships between cell division and fat cell cluster formation. Exp. Cell Res., 142, 273-281. 
GREEN H., KEHINDE O., 1975. An established preadipose cell line and its differentiation in culture. II. Factors affecting the adipose conversion. Cell, 5, 19-27.

GRIMALDI P., NEGREL R., AILHAUD G., 1978. Induction of the triglyceride pathway enzymes and of lipolytic enzymes during differentiation in a "preadipocyte" cell line. Eur. J. Biochem., 84, 369-376.

GRIMALDI P., NEGREL R., VINCENT J. P., AILHAUD G., 1979. Differenciation of Ob17 preadipocytes to adipocytes. Effect of insulin on the levels of insulin receptors and on the transport of $\alpha$-aminoisobutyrate. J. biol. Chem., 254, 6849-6852.

GRIMALDI P., DJIAN P., NEGREL R., AILHAÚD G., 1982. Differentiation of Ob17 preadipocytes to adipocytes : requirement of adipose conversion factor(s) for fat cell cluster formation, EMBO J., 1, 687-692.

KING G. L., KAHN C. R., RECHLER M. M., NISSLEY S. P., 1980. Direct demonstration of separate receptors for growth and metabolic activities of insulin and multiplicationstimulating activity (an insulin-like growth factor) using antibodies to the insulin receptor. J. clin. Invest., 66, 130-140.

NĖGREL R., GRIMALDI P., AILHAUD G., 1978. Establishment of preadipocyte clonal cell line from epididymal fat pad of ob/ob mouse that respond to insulin and to lipolytic hormones. Proc. nat. Acad. Sci. USA, 75, 6054-6058. 